\title{
The Behavior of Water Confined in Chemically Deteriorated PEFC Membranes as Studied by ${ }^{1} \mathrm{H}$ NMR Spectroscopy
}

\author{
Atsuko Y. Nosaka, ${ }^{a *}$ Satoru Watanabe, ${ }^{\mathrm{b}}$ Ichiro Toyoda, ${ }^{\mathrm{b}}$ and Yoshio Nosaka ${ }^{\text {* }}$ \\ ${ }^{a}$ Department of Chemistry, Nagaoka University of Technology (1603-1, Kamitomioka, Nagaoka 940-2188, Japan) \\ b Advanced Technology Research Center, Mitsubishi Heavy Industries, LTD. (1-8-1, Sachiura, Yokohama 236-8515, \\ Japan)
}

Received July 28, 2006 ; Accepted August 28, 2006

\begin{abstract}
The deterioration of the solid polymer electrolyte membrane employed for the polymer electrolyte fuel cells (PEFCs) during the operations is the urgent problem to be solved for the practical use of PEFCs. In the present study, to investigate the deterioration mechanisms, the morphological change of the membrane which was deteriorated chemically in Fenton media was studied by observing the spectral change of the confined water molecules in the membrane by ${ }^{1} \mathrm{H}$ NMR spectroscopy. For untreated membranes, the polymer structure is thermally converted at around $358 \mathrm{~K}$, so that the mobility of water became higher and the chemical environments became more homogeneous. However, it was revealed that the deteriorated membrane could not take such a specific structure that enables the higher conductivity of the membrane.
\end{abstract}

Key Words : Fuel Cell, Perfluorinated Ionomer Membrane, Deterioration, Water, ${ }^{1} \mathrm{H}$ NMR

\section{Introduction}

Because of the potential to provide a clean alternative to fossil fuels, intensive researches have been proceeding on the development of fuel cell systems. ${ }^{1,2)}$ Durability is one of the most concerns for the practical use of polymer electrolyte fuel cells (PEFCs). For this purpose, one of the most urgent technical problems that should be overcome is to prevent the solid polymer electrolyte membrane employed for PEFCs from the deterioration during the operations. Various mechanisms are being considered for the deterioration. ${ }^{3-10}$ ) The formation of reactive oxygen species during catalytic process in PEFC operation has been amply demonstrated. ${ }^{3)} \mathrm{H}_{2} \mathrm{O}_{2}$ and $\mathrm{OH}$ radical are the most commonly reported species and their presence is implicated in membrane deterioration. The membrane used for PEFCs is a proton-exchange membrane such as Nafion, which is the ionomer produced by DuPont consisting of a perfluoriated backbone and pendant chains terminated by sulfonic groups.

NMR is one of the most powerful techniques to investigate the molecular dynamics and structures to characterize the ionomers. ${ }^{1} \mathrm{H}-\mathrm{NMR}$ of water molecules adsorbed on solid materials often sensitively reflects the characteristics of the underlying materials. ${ }^{11-14)}$ Recently, we found that ${ }^{1} \mathrm{H}$ NMR signals of water confined in Nafion membranes presented characteristic changes after heating at $358 \mathrm{~K}^{14}{ }^{14}$ The phenomenon was explained by that the polymer morphology was thermally converted so that the chemical environments of water molecules became homogeneous and water mobility became high. The structure is considered to be stabilized most likely by the interaction of water molecules. In the present study, we investigated the spectral changes of the confined water in the chemically deteriorated membrane associated with the morphological change, and compared with those in the untreated membrane.

\section{Experimental}

Commercially available Nafion 112 membrane (E. I. duPont de Nemours and Co.) was used as received (the extent of swelling is not known). In the laboratory, $\mathrm{OH}$ radicals can be produced by Fenton reaction. ${ }^{15}$ The chemically decomposed membrane was prepared by treating Nafion 112 in Fenton media $\left(\mathrm{Fe}^{2+}\right.$ and $\mathrm{H}_{2} \mathrm{O}_{2}$ (30\%)) as follows. The membrane was immersed in $0.3 \mathrm{M}$ $\mathrm{FeSO}_{4}$ solution for $2 \mathrm{~h}$ under stirring. Then, it was soaked in a $30 \%(\mathrm{v} / \mathrm{v}) \mathrm{H}_{2} \mathrm{O}_{2}$ /water solution and kept at $343 \mathrm{~K}$ for $10 \mathrm{~h}$ under stirring. To eliminate $\mathrm{Fe}^{2+}$, the treated membrane was immersed in $1 \mathrm{M} \mathrm{HCl}$ solution for one night under stirring, and washed with distilled water to eliminate $\mathrm{Cl}$. After the treatements, the weight of the membrane was reduced by $9 \%$.

The ${ }^{1} \mathrm{H}$ NMR spectra of the membrane were measured in the form of a single sheet. The membranes were stored in ambient conditions, and were not subjected to any pretreatment before NMR measurements. A membrane sheet of $2 \times 50 \mathrm{~mm}$ was located in a $3 \mathrm{~mm}$ o.d. (55 $\mathrm{mm}$ high) glass NMR sample tube without adding an excess of water, which was capped and carefully sealed with Teflon films to keep it airtight. ${ }^{1} \mathrm{H}$ NMR measurements were carried out on a Varian Infinity Plus 500 NMR spectrometer at $500 \mathrm{MHz}$ in the temperature range of 297-358 K without magic angle spinning. The spectra were obtained after Fourier transformation of the FID signals following a single pulse excitation with pulse duration of $3.0 \mu \mathrm{s}$ and a $5 \mathrm{~s}$ relaxation delay. To eliminate the background signal of the NMR probe, the spectrum measured with an empty NMR sample tube was subtracted from all the spectra. Chemical shifts 
were measured relative to DSS (sodium2,2-dimethyl-2silapentane-5-sulfonate) as an external reference.

\section{Results and Discussion}

The ${ }^{1} \mathrm{H}$ NMR spectrum of water molecules in a hydrated Nafion 112 membrane in the form of a single sheet presented broad resonance lines as shown in Fig. 1A (a) at $297 \mathrm{~K}$. The resonance line was found to be apparently broadened by the distribution of chemical shifts of the water with different structures and mobility in the chemically different environments of the polymer based on the field dependency of the line width, and the line width is dependent on the humidification state of the membrane. ${ }^{14)}$ The chemical exchange among the water molecules in different chemical environments is considered to be slow in NMR time scale. The heterogeneity of the signals of the water suggests the heterogeneity of the polymer morphology and/or structure.

When the sample was heated at high temperature of $358 \mathrm{~K}$ in an open system, the water molecules were rapidly evaporated and NMR signal intensity was substantially reduced. On the other hand, as reported previously, ${ }^{14)}$ when the sample was heated gradually in a closed system under airtight conditions, the signal showed characteristic temperature dependence as shown in Fig. 1A. On increase of temperature, the signal shifted slightly upfield and the line width became sharper. The line width was reduced to $1 / 3$ of that before temperature increase when the sample was kept at $358 \mathrm{~K}$ for $1 \mathrm{~h}$ (Fig. 1A (c)). The line width remained sharp after the temperature was decreased back to $297 \mathrm{~K}$ (Fig. 1A (e)). The spectral feature held at least 18 days at $297 \mathrm{~K}$. On increase of temperature, the resonance line must become sharper partly because of the mobility gained. Taking into account that the resonance line is determined by the chemical shift distribution, the reduction of the line width could be also attributed to the reduced inhomogeneity of the chemical environments of water molecules. This would mean that the polymer structure was thermally converted so that the chemical environments of water molecules became homogeneous and/or chemical exchange among water molecules became rapid. ${ }^{14)}$ On the basis of the SANS (small angle scattering with neutrons) data of hydrated Nafion, Rollet et al. proposed polymeric aggregates surrounded by ionic groups and water molecules, contrary to the generally accepted inverse micelle type structure. ${ }^{16)}$ On increase of temperature the aggregates may resolve slowly to convert to the more homogeneous state. In this procedure, the bulk water spreads in the whole membrane, which enables the rapid chemical exchange among the water molecules in the whole membranes and enhances the mobility of water, resulting in the reduction of the line width of the water signal. The fact that the resonance line remained sharp after the temperature reverted to $297 \mathrm{~K}$ suggests that the converted polymer structure should be stable at $297 \mathrm{~K}$. Once the polymer takes the specified structure, it must be stabilized most likely by the interaction of water molecules since the phenomena were not observed under less humidification condi- tions. This would mean that in the presence of enough amounts of water molecules to stabilize the structure, the polymer could hold the homogeneous structure at $297 \mathrm{~K}$. The ionic conductivity of the membrane is closely related to the mobility of the water molecules in the membranes. ${ }^{17}$ ) The attained structure is considered to be favored to achieve the high ion conductivities of the membranes.

On the other hand, for the chemically deteriorated membrane, with increasing temperature, the signal shifted to the down field and the line width became broader. On decrease of temperature, the chemical shift reverted to the upfield, but the line width remained broad. The sample was kept at $328 \mathrm{~K}$ for $17 \mathrm{~h}$ but no significant change was observed. Further decrease of temperature to $297 \mathrm{~K}$, the sample showed almost the same spectral feature as that at $328 \mathrm{~K}$. These spectral changes clearly indicate that the deteriorated membrane did not undergo such morphological change as the untreated membrane within the temperature range studied. On increase of temperature, the mobility of water molecules must become higher, leading to the reduced line width. Therefore, taking into the account that the resonance line is determined by the distribution of chemical shifts, the observed line broadening would be ascribed to the contribution of the slow chemical exchange to the line width. At $358 \mathrm{~K}$ due to the evaporation of some amount of water from the membrane, the peak area of the water was reduced by about $20 \%$ as compared to those before temperature increase for both treated and untreated membranes. Since the bulk water is more volatile, the reduction of the peak area would mean mainly the evaporation of the certain amount of the bulk water from the membrane. Under this hydrated condition, the untreated membrane underwent the thermal conversion to the highly homogeneous morphology. Consequently, the

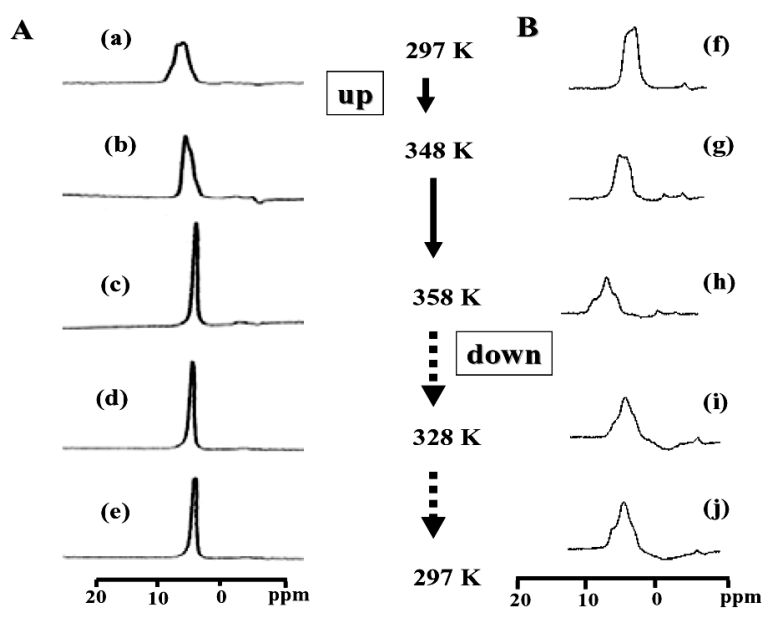

Fig. 1 Temperature dependence of ${ }^{1} \mathrm{H}-\mathrm{NMR}$ spectra of the water molecules confined in Nafion 112 (A), and the chemically deteriorated Nafion 112 (B).The spectra were measured initially at (a) (f); $297 \mathrm{~K}$ and the temperature was increased to (b) (g); $348 \mathrm{~K}$, and (c) (h); $358 \mathrm{~K}$, and then the temperature was decreased to (d) (i); $328 \mathrm{~K}$ and (e) (j); $297 \mathrm{~K}$. 
chemical environment of the confined water molecules would become also homogeneous, and the mobility and the chemical exchange among the water molecules were enhanced, resulting in the narrowing of the line width. On the other hand, for the deteriorated membrane, such a morphological conversion would not take place as suspected from the spectral change. For this case, the reduction of the bulk water in the membrane could cause the localization of the water around the separate polymer segments such as hydrophilic sulfonate groups, and hydrophobic fluorocarbon main and side chains. By measuring the IR spectra of $\mathrm{H}_{2} \mathrm{O}, \mathrm{D}_{2} \mathrm{O}$ and $\mathrm{HDO}$ in hydrated Nafion membranes, Falk reported that a substantial proportion of water molecules appeared to have an $\mathrm{OH}$ group (or occasionally both $\mathrm{OH}$ groups) exposed to the fluorocarbon environment. ${ }^{18)}$ Thus, the chemical exchange among the water molecules inter the distinct domains is considered to become slow in the NMR time scale and the signal became apparently broadened. In these local environments, the water molecules form a cluster which is stabilized by strong hydrogen bonding among the water molecules, leading to chemical shift to the lower field. Besides the localization of water molecules, the reduction of the bulk water molecules appeared to cause the change in the morphology of the deteriorated membrane because the spectral feature did not recover even after the temperature reverted from 358 to $297 \mathrm{~K}$. Thus, the lack of the specific position would prevent the deteriorated polymer from taking the favorable morphology or the structure in which water molecules are highly mobile to enable the high conductivity.

From both spectroscopic and thermal analyses, Grava et al. showed that $\mathrm{H}_{2} \mathrm{O}_{2}$ treatment changed the perfluorosufonated ionomer polymer structures and mechanical properties. From PAS-IR spectral profile they suggested the possibility of the formation of a crosslinking S-O-S between two side groups, as a result of the oxidation provoked by the hydrogen peroxide. ${ }^{9)}$ On the basis of the results of FT-IR and ${ }^{19} \mathrm{~F}$ NMR measurements of deteriorated Nafion membrane in $\mathrm{H}_{2} \mathrm{O}_{2}$ and $\mathrm{Fe}^{2+}$ media, Kinumoto et al. suggested that both the main and side chains were decomposed at similar rates by radical attack, most probably because the decomposition proceeded through radical de-polymerization (so-called unzippering mechanism). ${ }^{10}$

The loss of sulfonic acid groups of Nafion reduces the proton conductivity, and the decomposition of the C-F bonds leads to membrane thinning and the formation of pinholes. The present results also indicate the possibility that the lack of the specific position in the chemically deteriorated membrane make the polymer unable to take the morphology or the structure in which water molecules are highly mobile to enable the high conductivity. Consequently, the conductivity would fall during the practical operation.

\section{Conclusion}

In order to obtain the clue on the detailed deterioration mechanisms of the solid polymer electrolyte mem- brane employed for PEFCs, the chemically deteriorated Nafion 112 membrane was prepared in Fenton media. The ${ }^{1} \mathrm{H}$ NMR spectral changes of the confined water molecules in the deteriorated membrane after heating up to $358 \mathrm{~K}$ were compared with those in the untreated membrane. For the untreated Nafion 112 membrane, the polymer structure is thermally converted at around 358 $\mathrm{K}$, so that the mobility of water becomes higher and the chemical environments become more homogeneous, which enables the higher conductivity of the membrane. However, it was indicated that in the deteriorated membrane, due to the lack of the specific position in the polymer, such a structural change did not take place. The preventions of the persistent changes in the morphology might be responsible for the lowering of the conductivity at the early stage of the deterioration during the practical operation.

\section{Acknowledgement}

This work was supported by Research and Development of Polymer Electrolyte Fuel Cells from New Energy and Industrial Technology Development Organization (NEDO), Japan.

\section{References}

1) K. Miyatake and M. Watanabe, Electrochemistry, 73, 12 (2005).

2) O. Savadogo, J. Power Sources, 127, 135 (2004).

3) A. Bosnjakovic and S. Schlik, J. Phys. Chem. B, 108, 4332 (2004).

4) A. Pozio, R. F. Silva, M. De Francesco, and L. Giorgi, Electrochim. Acta, 48, 1543 (2003).

5) D. Knight, K. M. Colbow, J. St-Pierre, and D. P. Wilkinson, J. Power Sources, 127, 127 (2004).

6) M. Aoki, H. Uchida, and M. Watanabe Electrochem. Commun., 7, 1434 (2005).

7) J. Xie, D. L. Wood III, K. L. More, T. A. Zawodzinski, P. Atanassov, and R. L. Borup, J. Electrochem. Soc., 152, A104 (2005).

8) J. Xie, D. L. Wood III, K. L. More, P. Atanassov, and R. L. Borup, J. Electrochem. Soc., 152, A1011 (2005).

9) W. M. Grava, T. Okada, and Y. Kawano Electrochemistry, 74, 467 (2006).

10) T. Kinumoto, M. Inaba, Y. Nakayama, K. Ogata, R. Umebayashi, A. Tasaka, Y. Iriyama, T. Abe, and Z. Ogumi, J. Power Sources, 158, 1222 (2006).

11) A. Y. Nosaka, E. Kojima, T. Fujiwara, H. Yagi, H. Akutsu, and Y. Nosaka, J. Phys. Chem. B, 107, 12042 (2003).

12) A. Y. Nosaka, T. Fujiwara, H. Yagi, H. Akutsu, and Y. Nosaka, J. Phys. Chem. B, 108, 9121 (2004).

13) A. Y. Nosaka, J. Nishino, T. Fujiwara, T. Ikegami, H. Yagi, H. Akutsu, and Y. Nosaka, J. Phys. Chem. B, 110, 8380 (2006).

14) A. Y. Nosaka, S. Watanabe, I. Toyoda, and Y. Nosaka, Macromolecules, 39, 4425 (2006).

15) C. Walling, Acc. Chem. Res., 8, 125 (1975).

16) A.-L. Rollet, O. Diat, and G. Gebel, J. Phys. Chem. B, 106, 3033 (2002).

17) S. J. Paddison, R. Paul, and T. A. Zawodzinski, Jr, J. Electrochem. Soc., 147, 617 (2000).

18） M. Falk, Can. J. Chem., 58, 1495 (1980). 